\title{
Multifrequency Instability of Cavitating Inducers
}

\section{Christopher E. Brennen California Institute of Technology, 1201 East California Boulevard, Pasadena, CA 91125}

Recent testing of high-speed cavitating turbopump inducers has revealed the existence of more complex instabilities than the previously recognized cavitating surge and rotating cavitation. This paper explores one such instability that is uncovered by considering the effect of a downstream asymmetry, such as a volute on a rotating disturbance similar to (but not identical to) that which occurs in rotating cavitation. The analysis uncovers a new instability that may be of particular concern because it occurs at cavitation numbers well above those at which conventional surge and rotating cavitation occur. This means that it will not necessarily be avoided by the conventional strategy of maintaining a cavitation number well above the performance degradation level. The analysis considers a general surge component at an arbitrary frequency $\omega$ present in a pump rotating at frequency $\Omega$ and shows that the existence of a discharge asymmetry gives rise not only to beat components at frequencies, $\Omega-\omega$ and $\Omega+\omega$ (as well as higher harmonics), but also to rotating as well as surge components at all these frequencies. In addition, these interactions between the frequencies and the surge and rotating modes lead to "coupling impedances" that effect the dynamics of each of the basic frequencies. We evaluate these coupling impedances and show not only that they can be negative (and thus promote instability) but also are most negative for surge frequencies just a little below $\Omega$. This implies potential for an instability involving the coupling of a surge mode with a frequency around $0.9 \Omega$ and a low-frequency rotating mode about $0.1 \Omega$. We also examine how such an instability would be manifest in unsteady pressure measurements at the inlet to and discharge from a cavitating pump and establish a "footprint" for the recognition of such an instability. [DOI: 10.1115/1.2734238]

\section{Introduction}

Ever since the recognition of the POGO instability of liquidpropelled rockets more than 40 years ago $[1,2]$, much attention has been directed at understanding the mechanisms for the promotion of instabilities associated with cavitating pumps. The simple forms of POGO, of cavitation surge and of rotating cavitation, have been successfully studied and related to dynamic features in the performance of pumps such as the cavitation compliance and mass flow gain factor [3].

However, recent experience in several space programs around the world has indicated that the preceding body of knowledge and understanding may not be adequate or sufficient. Specifically, there appear to be other, more complex instabilities associated with cavitating turbopumps that do not fit easily within the current understanding. This paper explores one particular complex instability which is uncovered by considering the effect of a downstream asymmetry, such as a volute on a rotating disturbance similar to (but not identical to) that which occurs in rotating cavitation. The analysis which follows uncovers a new instability which may be of particular concern because it occurs at cavitation numbers well above those at which conventional surge and rotating cavitation occur. This means that it will not necessarily be avoided by the conventional strategy of maintaining a cavitation number well above the performance degradation level.

\section{Analysis}

As is conventional [3], we consider unsteady linear perturbations in the total pressure $p$ and mass flow rate $m$ given by

$$
p_{i}(t)=\bar{p}_{i}+\operatorname{Re}\left\{\widetilde{p}_{i, \omega} e^{j \omega t}\right\}
$$

Contributed by the Fluids Engineering Division of ASME for publication in the Journal of Fluids EngineERING. Manuscript received August 28, 2006; final manuscript received December 21, 2006. Review conducted by Akira Goto.

$$
m_{i}(t)=\bar{m}_{i}+\operatorname{Re}\left\{\tilde{m}_{i, \omega} e^{j \omega t}\right\}
$$

where $j$ is $(-1)^{1 / 2}$, the index $i$ denotes a specific location in the fluid flow path, the overbar denotes a mean or time-averaged quantity, a tilde denotes a fluctuating quantity, and Re denotes the real part. Implicit in the above linearization is a summation of the fluctuating terms over all the frequencies $\omega$ of interest. Thus, the complex quantities $\widetilde{p}_{i, \omega}$ and $\widetilde{m}_{i, \omega}$ incorporate the amplitude and phase of the fluctuating total pressure and mass flow rate at the frequency $\omega$ and the location $i$.

It is both convenient and, nowadays, conventional (see [4]) to express the dynamic response of a cavitating pump at the frequency $\omega$ by the relations

$$
\begin{aligned}
& \tilde{p}_{2, \omega}-\tilde{p}_{1, \omega}=G \tilde{p}_{1, \omega}-(R+j \omega L) \tilde{m}_{2, \omega} \\
& \tilde{m}_{2, \omega}-\tilde{m}_{1, \omega}=-j \omega C \widetilde{p}_{1, \omega}-j \omega M \tilde{m}_{1, \omega}
\end{aligned}
$$

where the subscripts $i=1$ and $i=2$ refer to the pump inlet and discharge locations and $G+1, R, L, C$, and $M$ are known respectively, as the pump gain, resistance, inertance, compliance, and mass flow gain factor. (Note the above differs slightly from the original form proposed by Brennen and Acosta [5] and used by $\mathrm{Ng}$ and Brennen [6] and Brennen et al. [7], Brennen [8], and Rubin [9], in measuring and analyzing the pump dynamic characteristics in that $\widetilde{m}_{2, \omega}$ rather than $\widetilde{m}_{1, \omega}$ is used at the end of Eq. (3). However, one form is readily transposed to the other.) Implicit in the above model (Eqs. (3) and (4)) is the assumption of linear relations; we note that although nonlinear effects undoubtedly occur, they are currently beyond our ability to analyze.

In this paper, we focus on the following issue originally suggested by Rubin [9]. The flow through the pump rotor consists of the sum of the flows through each of the blade passages. If all blade passages were identical at all moments in time, we could write the corresponding transfer function for each of the blade passages (say $K$ in number) as

$$
\widetilde{p}_{2, \omega, k}-\widetilde{p}_{1, \omega, k}=G \widetilde{p}_{1, \omega, k}-(R+j \omega L) K \widetilde{m}_{2, \omega, k}
$$




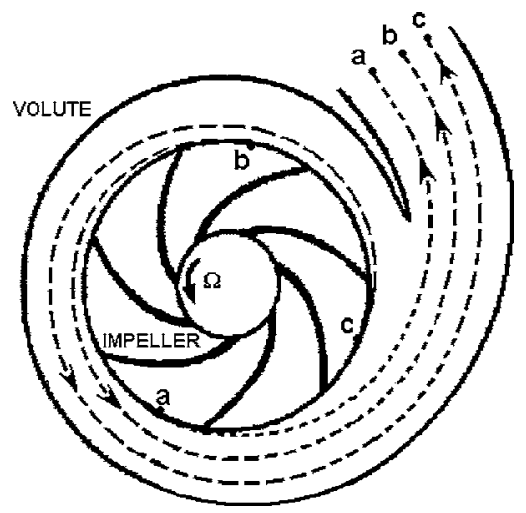

Fig. 1 Sketch of a pump volute showing typical streamlines $a a, b b$, and $c c$ with different path lengths

$$
\widetilde{m}_{2, \omega, k}-\widetilde{m}_{1, \omega, k}=-j \omega \frac{C}{K} \widetilde{p}_{1, \omega, k}-j \omega M \tilde{m}_{1, \omega, k}
$$

where the additional index $k=1,2, \ldots, K$ denotes the total pressures and mass flow rates in each of the $K$ blade passages. If this were all the dynamics to be concerned with, then, clearly, Eqs. (5) and (6) sum to yield the overall transfer function for the pump given by Eqs. (3) and (4), where

$$
\begin{gathered}
\tilde{p}_{2, \omega, k}=\tilde{p}_{2, \omega} ; \quad \tilde{p}_{1, \omega, k}=\tilde{p}_{1, \omega} \\
\tilde{m}_{2, \omega}=\sum_{k=1}^{K} \tilde{m}_{2, \omega, k} ; \quad \tilde{m}_{1, \omega}=\sum_{k=1}^{K} \tilde{m}_{1, \omega, k}
\end{gathered}
$$

However, the effect that we wish to focus on here is the fact that the discharge from a pump is usually quite asymmetric. Often the discharging flow is collected in a single volute, such as that shown in Fig. 1, so that, at any instant in time, the flow exiting from one blade passage may have much farther to travel than the flow from another blade passage, as illustrated by the three typical streamlines $a a, b b$, and $c c$ in Fig. 1. Of course, inlet flows may also be quite asymmmetric. However, we should note the relative effects of inlet and discharge asymmetries on the flows through an inducer or impeller as investigated by Bhattacharyya [10]. Bhattacharyya found that inlet asymmetries have a remarkably small effect on the asymmetry of the flow through an inducer, even when that asymmetry consisted of an abrupt right-angle bend just upstream of the inducer inlet. On the other hand, asymmetries in the discharge flow had surprisingly strong effects on the asymmetry of the flow through the inducer. For this reason and for the added reason of simplicity, we confine ourselves here to asymmetries in the discharge.

The primary effect of simple discharge asymmetry, such as produced by a single volute or by a right-angle bend just downstream of the impeller, is that the effective length of each flow passage oscillates at the rotational frequency of the impeller $\Omega$. This means that the instantaneous inertance of each blade passage flow is oscillating at a frequency $\Omega$ and with some amplitude, which we will denote by $L^{*}$. Of course, the resistance will also oscillate but, for simplicity, we neglect this effect (it is also much easier to estimate the magnitude of $L^{*}$ ). This additional dynamic effect is then incorporated into our model of the pump, as shown in Fig. 2. Each blade passage now has the original dynamics as represented by Eqs. (5) and (6) but with $\widetilde{p}_{2, \omega, k}$ now replaced by $\widetilde{p}_{M, \omega, k}$, where the location $M$ is the exit from the impeller blade passage, $k$; thus,

$$
\tilde{p}_{M, \omega, k}-\widetilde{p}_{1, \omega, k}=G \widetilde{p}_{1, \omega, k}-(R+j \omega L) K \tilde{m}_{2, \omega, k}
$$

replaces Eq. (5), whereas Eq. (6) remains unchanged. As incorporated in Eq. (15), we then add the additional oscillating inertance $L^{* *}$ onto the original blade passage dynamics, where

$$
L^{* *}=\operatorname{Re}\left\{L^{*} e^{j \Omega t+j 2 \pi k / K}\right\}
$$

where $L^{*}$ is a real constant and the term $j 2 \pi k / K$ incorporates the appropriate phase between the blade passages. Note that the direction of this phase or time delay is consistent with an index or passage label $k$ that increases in the direction of rotation of the impeller. Note also that since

$$
\sum_{k=1}^{K} e^{j 2 \pi k / K}=0
$$

the arithmetic means of the blade passage pressures at discharge $(i=2)$ and at the location $i=M$ are identical.

For later use, we also note that in a given blade passage $k$, the fluctuating mass flow rate at any general frequency, $\xi$ (specifically $\omega, \Omega-\omega, \Omega+\omega$, or higher-order combinations as anticipated below), namely, $\widetilde{m}_{2, \xi, k}$, must neccessarily consist of a "surge" component, $\widetilde{m}_{2, \xi} / K$, which is identical and in phase for all blade passages plus a "rotating" component, $\widetilde{m}_{\phi, \xi} e^{j 2 \pi k / K}$, which is caused by the downstream asymmetry. The phase of this second component must vary with $k$ in the same manner as the oscillatory inertance while the "magnitude," $\tilde{m}_{\phi, \xi}$, is identical for all blade passages. Consequently,

$$
\tilde{m}_{2, \xi, k}=\frac{\tilde{m}_{2, \xi}}{K}+\tilde{m}_{\phi, \xi} e^{j 2 \pi k / K}
$$

for $\xi=\omega, \Omega, \Omega-\omega, \Omega+\omega, \ldots$

In addition, we can anticipate that the existence of fluctuating components at the frequecies $\omega$ and $\Omega$ will, through the fluctuating inertance, spawn additional components at combination frequencies $\Omega-\omega, \Omega+\omega$, as well as higher harmonics and higherorder combinations. Thus, it becomes necessary to include flow fluctuations at frequencies $\Omega,(\Omega-\omega)$, and $(\Omega+\omega)$ (and higher frequencies) as well as $\omega$. Therefore, the flow rate in an individual blade passage, $m_{2, k}(t)$, will need to be expressed by

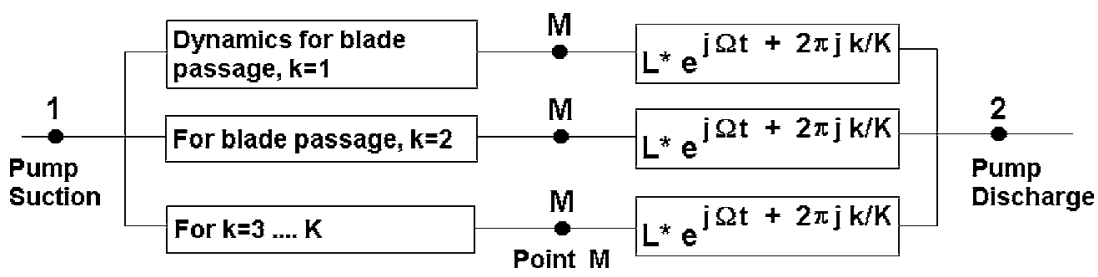

Fig. 2 Pump dynamic model with individual blade passage dynamics and an asymmetric discharge inertance 


$$
m_{2, k}(t)=\bar{m}_{2} / K+\operatorname{Re}\left\{\begin{array}{l}
\tilde{m}_{2, \omega, k} e^{j \omega t}+\tilde{m}_{2, \Omega, k} e^{j \Omega t} \\
+\tilde{m}_{2, \Omega-\omega, k} e^{j(\Omega-\omega) t}+\tilde{m}_{2, \Omega+\omega, k} e^{j(\Omega+\omega) t} \\
+\tilde{m}_{2,2 \Omega-\omega, k} e^{j(2 \Omega-\omega) t}+\tilde{m}_{2,2 \Omega+\omega, k} e^{j(2 \Omega+\omega) t} \\
+ \text { higher harmonics }
\end{array}\right\}
$$

Also, using the definition (1), the pressure difference, $p_{M, k}-p_{2}$, between the location $i=M$ and the discharge, $i=2$, should be similarly written as

$$
p_{M, k}-p_{2}=\bar{p}_{M}-\bar{p}_{2}+\operatorname{Re}\left\{\begin{array}{l}
\left(\widetilde{p}_{M, \omega, k}-\widetilde{p}_{2, \omega}\right) e^{j \omega t} \\
+\left(\widetilde{p}_{M, \Omega, k}-\widetilde{p}_{2, \Omega}\right) e^{j \Omega t} \\
+\left(\widetilde{p}_{M, \Omega-\omega, k}-\widetilde{p}_{2, \Omega-\omega}\right) e^{j(\Omega-\omega) t} \\
+\left(\widetilde{p}_{M, \Omega+\omega, k}-\widetilde{p}_{2, \Omega+\omega}\right) e^{j(\Omega+\omega) t} \\
+\left(\widetilde{p}_{M, 2 \Omega-\omega, k}-\widetilde{p}_{2,2 \Omega-\omega}\right) e^{j(2 \Omega-\omega) t} \\
+\left(\widetilde{p}_{M, 2 \Omega+\omega, k}-\widetilde{p}_{2,2 \Omega+\omega}\right) e^{j(2 \Omega+\omega) t} \\
+ \text { higher harmonics }
\end{array}\right\}
$$

Neglecting the resistance of the volute or elbow, this pressure difference must be equal to the inertance, $L^{* *}$, multiplied by the time derivative of the mass flow rate given by Eq. (13) so that

$$
p_{M, k}-p_{2}=L^{* *} \operatorname{Re}\left\{\begin{array}{l}
j \omega \tilde{m}_{2, \omega, k} e^{j \omega t}+j \Omega \tilde{m}_{2, \Omega, k} e^{j \Omega t} \\
+j(\Omega-\omega) \tilde{m}_{2, \Omega-\omega, k} e^{j(\Omega-\omega) t} \\
+j(\Omega+\omega) \tilde{m}_{2, \Omega+\omega, k} e^{j(\Omega+\omega) t} \\
+j(2 \Omega-\omega) \tilde{m}_{2,2 \Omega-\omega, k} e^{j(2 \Omega-\omega) t} \\
+j(2 \Omega+\omega) \tilde{m}_{2,2 \Omega+\omega, k} e^{j(2 \Omega+\omega) t}
\end{array}\right\}
$$

and substuting for $L^{* *}$ from Eq. (10), this becomes

$$
=\frac{L^{*}}{2} \operatorname{Re}\left\{\begin{array}{l}
j \Omega \tilde{m}_{2, \Omega, k} e^{-j 2 \pi k / K} \\
+j \Omega \tilde{m}_{2, \Omega, k} e^{2 j \Omega t+j 2 \pi k / K} \\
+j(\Omega+\omega) \tilde{m}_{2, \Omega+\omega, k} e^{j \omega t-j 2 \pi k / K} \\
-j(\Omega-\omega) \overline{\tilde{m}}_{2, \Omega-\omega, k} e^{j \omega t+j 2 \pi k / K} \\
+j \omega \tilde{m}_{2, \omega, k} e^{j(\Omega+\omega) t+j 2 \pi k / K} \\
-j \omega \overline{\tilde{m}}_{2, \omega, k} e^{j(\Omega-\omega) t+j 2 \pi k / K} \\
+j(\Omega-\omega) \tilde{m}_{2, \Omega-\omega, k} e^{j(2 \Omega-\omega) t+j 2 \pi k / K} \\
+j(\Omega+\omega) \tilde{m}_{2, \Omega+\omega, k} e^{j(2 \Omega+\omega) t+j 2 \pi k / K} \\
+j(2 \Omega-\omega) \tilde{m}_{2,2 \Omega-\omega, k} e^{j(\Omega-\omega) t-j 2 \pi k / K} \\
+j(2 \Omega+\omega) \tilde{m}_{2,2 \Omega+\omega, k} e^{j(3 \Omega-\omega) t+j 2 \pi k / K} \\
+ \text { higher harmonics }
\end{array}\right\}
$$

Thus, the introduction of the additional oscillatory inertance causes the generation of other fluctuating flow frequencies in addition to the basic frequency $\omega$ under consideration. Clearly, then, higher harmonics, such as $(3 \Omega-\omega),(3 \Omega+\omega)$, etc., may need to be included in the right-hand side of Eq. (14). However, since we will not pursue the solution for these higher frequencies, those terms will be dropped from further consideration here. For simplicity, we will retain only the terms that effect the frequencies $\omega$, $(\Omega-\omega)$, and $(\Omega+\omega)$. It follows from Eqs. (14) and (16) that, to first order,

$$
\begin{aligned}
\tilde{p}_{M, \omega, k}-\widetilde{p}_{2, \omega}= & -0.5 j(\Omega-\omega) L^{*} \overline{\tilde{m}}_{2, \Omega-\omega, k} e^{j 2 \pi k / K} \\
+ & 0.5 j(\Omega+\omega) L^{*} \tilde{m}_{2, \Omega+\omega, k} e^{-j 2 \pi k / K} \\
\widetilde{p}_{M, \Omega-\omega, k}-\widetilde{p}_{2,(\Omega-\omega)}= & -0.5 j \omega L^{*} \overline{\tilde{m}}_{2, \omega, k} e^{j 2 \pi k / K} \\
& +0.5 j(2 \Omega-\omega) L^{*} \tilde{m}_{2,2 \Omega-\omega, k} e^{-j 2 \pi k / K}
\end{aligned}
$$

$$
\begin{aligned}
\tilde{p}_{M, \Omega+\omega, k}-\tilde{p}_{2,(\Omega+\omega)}= & 0.5 j \omega L^{*} \tilde{m}_{2, \omega, k} e^{j 2 \pi k / K} \\
& +0.5 j(2 \Omega+\omega) L^{*} \tilde{m}_{2,2 \Omega+\omega, k} e^{-j 2 \pi k / K}
\end{aligned}
$$

where the additional overbar above the tilde denotes the complex conjugate.

Using the above relations, we can eliminate the intermediate pressures at the point $M$ from the transfer function Eqs. (9) for the frequencies $\omega,(\Omega-\omega)$, and $(\Omega+\omega)$. Then, we substitute the decomposition given in Eq. (12) and generate an array of relations for the various fluctuating pressures and mass flow rates by summing the resulting equations over the blade passages using the relations (7), (8), and (11). This yields

$$
\begin{aligned}
\tilde{p}_{2, \omega}-\tilde{p}_{1, \omega}=G \tilde{p}_{1, \omega}-(R+j \omega L) \tilde{m}_{2, \omega}+0.5 j(\Omega-\omega) L^{*} \overline{\tilde{m}}_{\phi, \Omega-\omega} \\
-0.5 j(\Omega+\omega) L^{*} \tilde{m}_{\phi, \Omega+\omega} \\
\tilde{p}_{2, \Omega-\omega}-\widetilde{p}_{1, \Omega-\omega}=G \widetilde{p}_{1, \Omega-\omega}-\{R+j(\Omega-\omega) L\} \tilde{m}_{2, \Omega-\omega} \\
+0.5 j \omega L^{*} \overline{\tilde{m}}_{\phi, \omega}-0.5 j(2 \Omega-\omega) L^{*} \tilde{m}_{\phi, 2 \Omega-\omega}
\end{aligned}
$$

$$
\begin{aligned}
\tilde{p}_{2, \Omega+\omega}-\widetilde{p}_{1, \Omega+\omega}= & G \tilde{p}_{1, \Omega+\omega}-\{R+j(\Omega+\omega) L\} \tilde{m}_{2, \Omega+\omega} \\
& -0.5 j(2 \Omega+\omega) L^{*} \tilde{m}_{\phi, 2 \Omega+\omega}
\end{aligned}
$$

Note the two new terms in the first and second transfer function equations and the one new term in the third and observe that these imply interactions between the frequencies. Specifically note how the fluctuating flow rate at the frequency $\omega$ feeds into the $(\Omega$ $-\omega)$ frequency but not the $(\Omega+\omega)$ frequency.

Additional important relations are obtained by multiplying each of the array of relations by $e^{-j 2 \pi k / K}$ or $e^{-j 2 \pi k / K}$ before summing. These manipulations yield relations for the $\tilde{m}_{\phi, \omega}$ rotating components of the fluctuating mass flow rates as follows:

$$
\begin{gathered}
\tilde{m}_{\phi, \omega}=\frac{j(\Omega-\omega) L^{*}}{2 K^{2}(R+j \omega L)} \overline{\tilde{m}}_{2, \Omega-\omega} \\
\tilde{m}_{\phi, \Omega-\omega}=\frac{j \omega L^{*}}{2 K^{2}\{R+j(\Omega-\omega) L\}} \overline{\tilde{m}}_{2, \omega} \\
\tilde{m}_{\phi, \Omega+\omega}=-\frac{j \omega L^{*}}{2 K^{2}\{R+j(\Omega+\omega) L\}} \tilde{m}_{2, \omega} \\
\tilde{m}_{\phi, 2 \Omega-\omega}=-\frac{j(\Omega-\omega) L^{*}}{2 K^{2}\{R+j(2 \Omega+\omega) L\}} \tilde{m}_{2, \Omega-\omega} \\
\tilde{m}_{\phi, 2 \Omega+\omega}=-\frac{j(\Omega+\omega) L^{*}}{2 K^{2}\{R+j(2 \Omega+\omega) L\}} \tilde{m}_{2, \Omega+\omega}
\end{gathered}
$$

and the following for the higher-order surge components:

$$
\tilde{m}_{2, \Omega+\omega}=\widetilde{m}_{2,2 \Omega-\omega}=\widetilde{m}_{2,2 \Omega+\omega}=\tilde{m}_{2,3 \Omega-\omega}=\tilde{m}_{2,3 \Omega+\omega}=0
$$

Note the symmetry in the relations (23) and (24).

Using the above relations $\widetilde{m}_{\phi, \omega}, \widetilde{m}_{\phi, \Omega-\omega}$ and $\widetilde{m}_{\phi, \Omega+\omega}$ can be eliminated from Eqs. (20)-(22) so to obtain the coupled transfer function equations for the frequencies $\omega$ and $\Omega-\omega$

$$
\begin{gathered}
\tilde{p}_{2, \omega}-\widetilde{p}_{1, \omega}=G \widetilde{p}_{1, \omega}-\left(R+j \omega L+X_{\omega}\right) \tilde{m}_{2, \omega} \\
\tilde{p}_{2, \Omega-\omega}-\widetilde{p}_{1, \Omega-\omega}=G \widetilde{p}_{1, \Omega-\omega}-\left\{R+j(\Omega-\omega) L+X_{\Omega-\omega}\right\} \widetilde{m}_{2, \Omega-\omega}
\end{gathered}
$$

$$
\tilde{p}_{2, \Omega+\omega}-\tilde{p}_{1, \Omega+\omega}=G \tilde{p}_{1, \Omega+\omega}-\left\{R+j(\Omega+\omega) L+X_{\Omega+\omega}\right\} \tilde{m}_{2, \Omega+\omega}
$$

where the "coupling impedances" 


$$
\begin{gathered}
X_{\omega}=\frac{\omega L^{* 2}}{2 K^{2}} \frac{\left\{\omega R-j\left(\Omega^{2}-\omega^{2}\right) L\right\}}{\{R-j(\Omega-\omega) L\}\{R+j(\Omega+\omega) L\}}=Z(\omega) \\
X_{\Omega-\omega}=\frac{(\Omega-\omega) L^{* 2}}{2 K^{2}} \frac{\{(\Omega-\omega) R-j \omega(2 \Omega-\omega) L\}}{\{R-j \omega L\}\{R+j(2 \Omega-\omega) L\}}=Z(\Omega-\omega) \\
X_{\Omega+\omega}=\frac{\omega L^{* 2}}{4 K^{2}} \frac{(\Omega+\omega)(2 \Omega-\omega)}{\{R+j(2 \Omega+\omega) L\}}
\end{gathered}
$$

Note, again, the symmetry inherent in the impedances, $X_{\omega}$ and $X_{\Omega-\omega}$, such that they may be represented by the single function, $Z(\omega)$, as defined on the right in the Eqs. (32)-(34).

\section{Pump Transfer Functions}

In conclusion, we have determined the pump transfer function equations, that should be used for the stability analyses for the fluctuating pressures and mass flow rates:

- For the general frequency $\omega$, Eqs. (29) (with Eq. (32) and (4)

- For the companion frequency, $\Omega-\omega$, Eqs. (30) (with Eq. (33) and (4) with $(\Omega-\omega)$ replacing $\omega$

The consequences of the interactive dynamics unveiled here, the actual stability analyses, will, of course, depend on the system within which the pump is operating. However, even without embarking on such a system-dependent analysis, we can investigate two consequences of the multifrequency transfer functions derived above; namely, we can examine:

- The pressures at various fixed circumferential locations upstream or downstream of the impeller since these are the commonly used diagnostic measurements that are made during testing

- The coupling impedances, $Z(\omega)$ and $Z(\Omega-\omega)$, since this function $Z$ is a primary factor effecting the stability of the system in which the pump might be installed

\section{Pressures at Fixed Locations}

Commonly, pressure transducers are installed at a number of fixed circumferential locations in order to observe and analyze the instabilities that occur within a cavitating inducer or pump. To examine the form of the pressure fluctuations that the above flow would cause at a fixed circumferential location close to the impeller, we choose to examine the pressures at the blade passage outlet location $i=M$. The pressure fluctuations at other axial locations close to the impeller (upstream or downstream) will have the same basic form and frequency components so confining attention to $i$ $=M$ does not limit the applicability of the results that follow.

First, we note that by using the expression (12) in Eqs. (17)-(19) (and using Eqs. (28)), the fluctuating pressures at the frequencies $\omega, \Omega-\omega$, and $\Omega+\omega$ in the frame of reference rotating with the impeller can be written in the following form:

$$
\begin{aligned}
& \operatorname{Re}\left\{\left(\tilde{p}_{M, \omega}^{\prime}-\frac{j(\Omega-\omega) L^{*}}{2 K} \overline{\tilde{m}}_{2, \Omega-\omega} e^{j 2 \pi k / K}\right) e^{j \omega t}\right\} \\
& \operatorname{Re}\left\{\left(\tilde{p}_{M, \Omega-\omega}^{\prime}-\frac{j \omega L^{*}}{2 K} \overline{\tilde{m}}_{2, \omega} e^{j 2 \pi k / K}\right) e^{j(\Omega-\omega) t}\right\} \\
& \operatorname{Re}\left\{\left(\tilde{p}_{M, \Omega+\omega}^{\prime}+\frac{j \omega L^{*}}{2 K} \tilde{m}_{2, \omega} e^{j 2 \pi k / K}\right) e^{j(\Omega+\omega) t}\right\}
\end{aligned}
$$

where the primes denote modifications in amplitude that are inconsequential to the conclusions of this section; similar expressions for the frequencies $2 \Omega-\omega$ and $2 \Omega+\omega$ are also needed. These fluctuations in pressure must then be translated into a fixed or nonrotating frame of reference. To do this, we define a set of nonrotating coordinates as follows: (i) an angular circumferential coordinate, $\theta^{*}=\Omega t+2 \pi k / K$ and (ii) time, $t^{*}=t$. One of the complications of this coordinate transformation is that the second terms in Eqs. (35)-(37) now need to be assigned to a different frequency. The resulting fluctuating pressures in the fixed coordinate frame at the frequencies $\omega, \Omega-\omega$, and $\Omega+\omega$ then take the following form:

$$
\begin{gathered}
\operatorname{Re}\left\{\left(\tilde{p}_{M, \omega}^{\prime}+\frac{j \omega L^{*}}{K} \cos \theta^{*} \tilde{m}_{2, \omega}\right) e^{j \omega t^{*}}\right\} \\
\operatorname{Re}\left\{\left(\tilde{p}_{M, \Omega-\omega}^{\prime}+\frac{j(\Omega-\omega) L^{*}}{K} \cos \theta^{*} \tilde{m}_{2, \Omega-\omega}\right) e^{j(\Omega-\omega) t^{*}}\right\} \\
\operatorname{Re}\left\{\left(\tilde{p}_{M, \Omega+\omega}^{\prime}\right) e^{j(\Omega+\omega) t^{*}}\right\}
\end{gathered}
$$

Consequently, the pressure fluctuations that would be observed at fixed, nonrotating circumferential locations near the impeller as a result of the flow constructed in the preceding sections would have the following features:

1. The fluctuating pressures at the basic instability frequency $\omega$ would consist of a surge mode whose amplitude varies from one circumferential location to another. It would contain no rotating component. For convenience of discussion, we will refer to this as the "surge" mode and frequency.

2. The fluctuating pressures at the frequency $\Omega-\omega$ would be observed to have two components: a surge component and a component that has a single cell that rotates in the direction of impeller rotation. Though it does contain a surge component we will, for convenience refer to this as the "rotating" mode and frequency.

3. A fluctuating pressure at the frequency $\Omega+\omega$ that has only a surge component (no rotating component).

4. Higher harmonics, $2 \Omega-\omega, 2 \Omega+\omega$, etc., and higher cell numbers (arising from terms with more than one circumferential cell).

This paper is focused on the interaction between the surge and rotating modes. We reserve comment on some measured pressure recordings until the end of Sec. 5 .

\section{Coupling Impedance}

Now examine the stability issue in more detail by focusing on the coupling impedance, $Z(\omega)$, defined in Eqs. (32) and (33) and whose consequences are imbedded in Eqs. (29) and (30). Note that $Z(\omega)$ is proportional to the fluctuating inertance squared, $L^{* 2}$. Indeed, the presence of the ratio $L^{*} / K$ means that this coupling impedance, $Z(\omega)$, is proportional to the square of the inertance of the entire flow in the mixing section downstream of the impeller discharge and before the end of the asymmetry (since inertance is inversely proportional to the cross-sectional area of the flow). In the analysis that follows, this factor appears as the parameter $L^{*} / K L$ or the ratio of the amplitude of the fluctuating inertance to the main pump inertance.

Next, we examine the variation of the coupling impedance over the range of instability frequencies, $0<\omega<\Omega$, in order to evaluate its consequences. From Eqs. (29) and (30), we observe that if the real part of the coupling impedance were to become sufficiently negative so that it overcame the pump resistance $R$ and led to a negative total resistance of the pump, $R+\operatorname{Re}\{Z(\omega)\}$, then instability at that frequency $\omega$ would be a likely consequence. To investigate this further, we examine the real part of the coupling impedance, $\operatorname{Re}\{Z(\omega)\}$, which, from Eq. (32), can be written as

$$
\frac{\operatorname{Re}\{Z(\omega)\}}{R}=\frac{1}{2}\left\{\frac{L^{*}}{K L}\right\}^{2} Z^{*}(\xi)
$$

where $\xi=\omega / \Omega$ and $Z^{*}$ is defined as 


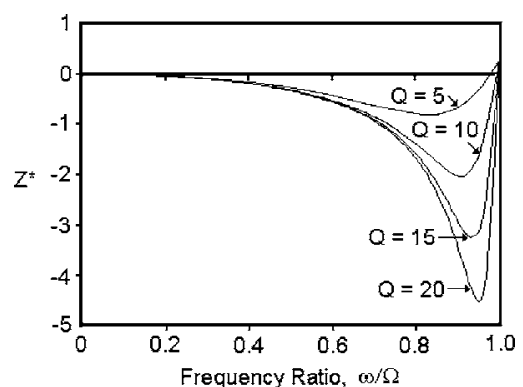

Fig. 3 Coupling resistance function $Z \subset$ plotted against the frequency ratio, $\omega / \Omega$, for four values of $Q=\Omega L / R$

$$
Z^{*}(\xi)=\frac{\xi^{2}\{\Omega L / R\}^{2}\left[1+\left(\xi^{2}-1\right)\{\Omega L / R\}^{2}\right]}{\left[1+(1-\xi)^{2}\{\Omega L / R\}^{2}\right]\left[1+(1+\xi)^{2}\{\Omega L / R\}^{2}\right]}
$$

Typical values of $Z^{*}(\omega / \Omega)$ are presented in Fig. 3 for various typical values of the parameter $\Omega L / R$. Note that $Z^{*}$ is negative over most of the range $0<\omega / \Omega<1$. This negative value could cause $R+\operatorname{Re}\{Z(\omega)\}$ to become negative. Consequently, the potential for instability exists though the outcome depends on the magnitude of $L^{*} / K L$, on the parameter $\Omega L / R$ and on the frequency $\omega$.

In Fig. 3, we have chosen to present results for a range of values of $\Omega L / R$. Estimates of the magnitude of this parameter from measurements of the dynamics of a particular cavitating inducer by $\mathrm{Ng}$ and Brennen [6] and Brennen et al. [7] (see also [4]) yield values of $\Omega L / R$ that range between about 2 and 20 . As another data point we note that a typical value for a centrifugal pump from the measurements of Anderson et al. [11] is $\sim 6$. Within this range of $\Omega L / R$, it is clear from Fig. 3 that the most highly negative values of the coupling resistance occur for values of $\omega$ just a little less than $\Omega$ and in the neighborhood of $0.9 \Omega$. Thus the instability that is most likely is one with a surge mode at a frequency a little less than $\Omega$ (say $0.9 \Omega$ ) coupled with a rotating mode with a low frequency like $0.1 \Omega$.

We might also ask whether the magnitude of $\operatorname{Re}\{Z(\omega)\}$ could approach the magnitude of $R$ so that $R+\operatorname{Re}\{Z(\omega)\}$ could become negative. From Eq. (41), this depends on both $Z^{*}$ and $L^{*} / K L$. From Fig. 3, the magnitude of $Z^{*}$ can clearly be greater than unity. Moreover, Anderson et al. [11] measured separately the inertances of the impeller $(L)$ and of the volute $\left(L^{*} / K\right)$ for a typical centrifugal pump and found them to be of similar magnitude. Thus, at least in this single example, the magnitude of $L^{*} / K L$ could be of order unity. Consequently, in the most unstable range of frequency around $0.9 \Omega$, it is quite possible for $-\operatorname{Re}\{Z(\omega)\}$ to exceed $R$ and to cause instability.

In summary, the analysis predicts that if such an coupled instability were to occur for $\Omega L / R$ in the range of $2-20$, it would consist of a surge component at a frequency of about $0.9 \Omega$ coupled with a rotating component at a frequency of about $0.1 \Omega$. Unlike the conventional rotating cavitation or cavitation surge this instability is not a dynamic instability occurring where the quasistatic pump resistance is positive (and therefore requiring the dynamic cavitation characteristics, such as the mass flow gain factor for its onset) but rather a more basic quasistatic instability similar to compressor stall [3] in which the effective resistance becomes negative. It therefore does not depend directly on cavitation, though it is likely that cavitation compliance and mass flow gain factor will enter the analysis when the system response is included in the analysis and that cavitation is likely to encourage the onset of the instability by allowing flexibility for the growth of the surge component. But the bottom line is that this coupled instability, being relatively independent of the cavitation number, could occur at much higher values of that parameter.
The instability characteristics described in the preceding paragraphs precisely correspond with an unusual instability experienced during a recent liquid rocket engine test program. Pressure transducers in water facility tests of that inducer demonstrated the presence of a surge component at $0.9 \Omega$ coupled with a rotating component at $0.1 \Omega$. Moreover, the instability developed at cavitation numbers of the order of $0.04-0.1$, well above the values at which cavitation surge or rotating cavitation might be expected. It was also a function of the flow coefficient, showing a particular tendency to develop at flow coefficients below the design value.

\section{Conclusions}

Recent experience with rocket engine turbopumps has demonstrated that there exist instability modes in cavitating pumps/ inducers that are more complex than the traditional rotating cavitaton and cavitation surge instabilities. This paper investigates the possibility of such an instability that involves the coupling of a surge mode and a rotating mode. The instability is triggered, in part, by asymmetry in the pump discharge that excites surge within the individual blade passages. It is shown that this leads to a "coupling impedance" that has a negative resistance over a range of frequencies and can therefore lead to a negative pump resistance, which could cause instability. Moreover, it is shown that in a likely parameter range for cavitating inducers, the most unstable case involves the coupling of a surge mode at about $0.9 \Omega$ with a rotating component at $0.1 \Omega$. Furthermore, since the instability does not depend essentially on the presence of cavitation (unlike the traditional cavitating surge) it could occur at significantly higher cavitation numbers than surge or rotating cavitation. All of the characteristics of this nontraditional instability described above were observed while conducting a recent series of liquid engine ground tests.

\section{Acknowledgment}

The author wishes to acknowledge the comments of Sheldon Rubin [9], who, many years ago, suggested that we should consider the dynamics inherent in the different parallel flow paths within a pump and volute system. I also wish to thank Tom Zoladz and the NASA George Marshall Space Flight Center for their support under Grant No. NAG8-1934.

$$
\begin{aligned}
& \text { Nomenclature } \\
& C=\text { pump cavitation compliance } \\
& G+1=\text { pump gain } \\
& j=(-1)^{1 / 2} \\
& k=1,2, \ldots K \text { denotes a particular blade passage } \\
& K=\text { number of impeller blade passages } \\
& L=\text { pump inertance } \\
& L^{*}=\text { fluctuating discharge inertance amplitude } \\
& L^{* *}=\text { fluctuating discharge inertance } \\
& m_{i}=\text { mass flow rate at the location, } i \\
& m_{i, k}=\text { mass flow rate in the blade passage } k \text { at the } \\
& \text { location } i \\
& \bar{m}_{i}=\text { time-averaged mass flow rate at the location } i \\
& \tilde{m}_{i, \omega}=\text { complex amplitude of the fluctuating mass } \\
& \text { flow rate at the location } i \text { and frequency } \omega \\
& \tilde{m}_{i, \omega, k}=\text { complex amplitude of the fluctuating mass } \\
& \text { flow rate at the location } i \text { in the impeller blade } \\
& \text { passage } k \text { and at frequency } \omega \\
& \tilde{m}_{i, \xi} / K=\text { surge component of the fluctuating mass flow } \\
& \text { rate at the location } i \text { and frequency } \xi \\
& \tilde{m}_{\phi, \xi} / K=\text { rotating component of the fluctuating mass } \\
& \text { flow rate at the frequency } \xi \\
& M=\text { pump mass flow gain factor } \\
& p_{i}=\text { total pressure at the location } i
\end{aligned}
$$


$p_{M, k}=$ total pressure in the blade passage $k$ at the location $M$

$\bar{p}_{i}=$ time-averaged total pressure at the location $i$

$\tilde{p}_{i, \omega}=$ complex amplitude of the fluctuating total pressure at the location $i$ and frequency $\omega$

$\tilde{p}_{i, \omega, k}=$ complex amplitude of the fluctuating total pressure at the location, $i$, in the impeller blade passage $k$ and at frequency $\omega$

$R=$ pump resistance

$\operatorname{Re}\{\}=$ denotes "the real part of"

$t=$ time

$X_{\xi}=$ coupling impedance for the frequency $\xi$

$Z(\xi)=X_{\xi}$

$Z^{*}(\omega / \Omega)=$ dimensionless coupling impedance function

$\theta^{*}=\Omega t+2 \pi k / K$

$\xi=$ denotes a general radian frequency

$\omega=$ radian frequency of fluctuation

$\Omega=$ radian frequency of impeller rotation

$-{ }_{\sim}$ denotes complex conjugate when placed above

\section{References}

[1] Rubin, S., 1966, 'Longitudinal Instability of Liquid Rockets Due to Propulsion Feedback (Pogo)," J. Spacecr. Rockets, 3(8), pp. 1188-1195.

[2] NASA, 1970, "Prevention of Coupled Structure-Propulsion Instability," NASA SP-8055.

[3] Brennen, C. E., 1994, Hydrodynamics of Pumps, Concepts ETI and Oxford University Press, London.

[4] Rubin, S., 2004, "An Interpretation of Transfer Function Data for a Cavitating Pump," 40th AIAA/ASME/SAE/ASEE Joint Propulsion Conf., Paper No. AIAA-2004-4025.

[5] Brennen, C. E., and Acosta, A. J., 1976, "The Dynamic Transfer Function for a Cavitating Inducer," ASME J. Fluids Eng., 98, pp. 182-191.

[6] Ng, S. L., and Brennen, C. E., 1978, "Experiments on the Dynamic Behavior of Cavitating Pumps," ASME J. Fluids Eng., 100(2), pp. 166-176.

[7] Brennen, C. E., Meissner, C., Lo, E. Y., and Hoffman, G. S., 1982, "Scale Effects in the Dynamic Transfer Functions for Cavitating Inducers," ASME J. Fluids Eng., 104, pp. 428-433.

[8] Brennen, C. E., 1978, "Bubbly Flow Model for the Dynamic Characteristics of Cavitating Pumps," J. Fluid Mech., 89(2), 223-240.

[9] Rubin, S., 1975, Personal communication.

[10] Bhattacharyya, A., 1994, "Internal Flows and Force Matrices in Axial Flow Inducers," Ph.D. thesis, California Institute of Technology.

[11] Anderson, D. A., Blade, R. J., and Stevens, W., 1971, "Response of a RadialBladed Centrifugal Pump to Sinusoidal Disturbances for Non-Cavitating Flow," NASA TN D-6556. 